\title{
CELLULAR ORGANISATION OF THE OPTIC NERVE AND THE IMPLICATIONS FOR OPTIC NEURITIS
}

\author{
ALASTAIR COMPSTON \\ Cambridge
}

\begin{abstract}
SUMMARY
Opportunities for studying growth, degeneration and repair in the central nervous system have altered over the last decade with the development of techniques for culturing neurones and glia and the availability of immunological or molecular markers that identify separate lineages and their progeny. Much pioneering work has been carried out in the rodent optic nerve but the principles that emerge are representative for other parts of the nervous system; development of neurones and glia may differ substantially in rats and man, so that assumptions must be made in extrapolating from properties of the rat optic nerve to diseases of the human central nervous system.
\end{abstract}

Macroglia consist of astrocytes and oligodendrocytes; microglia are thought to be derived from circulating macrophages that populate the nervous system during development and function thereafter as immunologically competent cells ${ }^{1}$; they present antigen, remove debris by phagocytosis and reshape glial-neuronal arrangements as the nervous system develops. Oligodendrocytes synthesise and maintain myelin sheaths in the central nervous system; unlike Schwann cells, which myelinate single axons in the peripheral nervous system, oligodendrocytes ensheathe short segments of several neighbouring axons. Astrocytes provide a physical structure for the nervous system, define the glial limiting membrane, contribute to formation of the blood brain barrier and contact the nodes of Ranvier.

Development in the nervous system depends to some extent on the intrinsic molecular machinery of the neuronal and glial lineages but their differentiation and disstribution are also much influenced by the environment in which growth is occurring. Although embryonic cells have a greater capacity for proliferation than their adult progeny, organisation in the nervous system depends critically on the availability of growth factors that attract migrating neurones and guide their developing axons.

Correspondence to: Professor Alastair Compston, University of Cambridge Clinical School, Addenbrooke's Hospital, Hills Road, Cambridge CB2 2QQ.
Extremely complex connections have to be established between nerve cells during development, and these must remain stable; amongst their other roles, differentiated astrocytes and oligodendrocytes are each inhibitory to axon growth and contribute stability to the mature nervous system. One consequence of this switch from plasticity to inhibition is that the adult nervous system loses the capacity for regneration but its very complexity makes the brain extremely vulnerable to injury and disease. One hope is that re-creating the growth factor environment of the developing brain in the damaged adult nervous system could provide a means for repair following demyelination, but it may also be necessary to combine this strategy with the replacement of defective cells in order fully to restore sophisticated neurological function (see 2 , for review).

Neurones and axons are subject to ischaemic, toxic, traumatic and degenerative injury. Other disorders of the adult human nervous system are characterised, in the acute phase, by perivascular infiltration of inflammatory cells and this seems to have a selective effect on glia; inflammatory brain disease leads to oligodendrocyte depletion, demyelination and astrocytic overgrowth but the extent to which these features are seen in individual patients and in the different demyelinating diseases depends on the duration and rapidity with which the inflammatory response has occurred.

\section{CELLULAR ORGANISATION OF THE OPTIC NERVE}

Immunological, molecular and cell biological studies have provided a sophisticated account of development in the central nervous system but there are points of controversy. These concern the stage at which stem cells become irreversibly committed to differentiation down either the neuronal or glial lineage, and when the two main macroglial (astrocyte and oligodendrocyte) cell types diverge; opinions therefore differ as to whether or not the neonatal and adult nervous system contain bipotential cells, capable of differentiating either into astrocytes or oligodendrocytres, and so reconstituting gliopaenic areas in which myelin is lost, saltatory conduction altered and neurological function impaired. 
As the neural tube forms, multipotential stem cells are found in the ventricular and subventricular zones around the lateral ventricles. It seems likely that at a very early stage, under the influence of environmental cues, these lose their bipotentiality and develop into cells which are committed either to the neuronal or glial lineage ${ }^{3}$. Most cells in the subventricular zone are neuronal and have a low rate of division, whereas the glial lineage has a greater capacity for mitosis and fewer cells are found in the germinal zones.

The most informative cell surface markers for these stages in development are GD3 and RAT401, both of which are lost with emergence of the neuronal lineage. The first conspicuous morphological change in glia is the appearance of radial cells which stretch in parralel arrays from the subventricular zone to the subpial brain surface ${ }^{4}$. These radial glia correspond to the protoplasmic astrocytes depicted by Cajal using silver stains and now defined by the presence of the antigens RAT401, RC1 and RC2. Contemporary morphological studies indicate that initially these are bipolar, with a short process connecting the cell body to the adjacent ventricular zone, and a longer process penetrating the developing cortical plate, reaching to the pial surface and terminating in the glial limiting membrane. Outward migration of the cell body then occurs, altering the relative length of the two processes; as movement occurs towards the cortex, the descending process is lost, the ascending one elaborates and the cell begins to resemble the mature astrocyte with randomly orientated processes and reduced expression of RAT401, $\mathrm{RC} 1$ and $\mathrm{RC} 1$ antigens. The radial network co-incides with neuronal histogenesis suggesting that this serves as a scaffold for nerve cells migrating from their germinal zones towards the developing cortex; this wave of neuronal growth is associated with inhibition of cell proliferation in the glial network. But as nerve cells switch from the phase of migration and distribution to the establishment of their synaptic connections, radial glia start to differente and develop into multipolar stellate cells.

These are the type 1 astrocytes that first populate the developing optic nerve and they are positive for the RAN2 and GFAP markers. Their density decreases from the eye to the brain end of the nerve. One view is that type 1 astrocytes act as a source of platelet derived growth factor (PDGF) which serves as a proliferative and chemotactic signal for the other main glial lineage, the oligodendrocyte-type 2 astrocyte $(0-2 \mathrm{~A})$ progenitor cell $^{5-7}$; this is defined by $\mathrm{A} 2 \mathrm{~B} 5$ and the presence of receptors for tetanus toxin and PDGF. The 0-2A progenitor proliferates and populates the optic nerve, migrating from tissues located around the optic chiasm and not differentiating until the time of birth. At about that time, the intrinsic proliferative potential of the neonatal 0-2A progenitor decreases and, with reduction in the secretion of PDGF by type 1 astrocytes $^{8}$, differentiation occurs down the constitutive pathway into oligodendrocytes; these synthesise cell processes that, in response to axonal signals, will constitute the myelin membrane.
One source of controversy in this account is the extent to which the growth factor environment drives proliferation and differentiation in the $0-2 \mathrm{~A}$ progenitor. The conventional view that oligodendrocyte differentiation occurs constitutively after a fixed number of progenitor divisions, occurring in response to PDGF, now has to be modified by the observations that, at least in vitro, basic fibroblast growth factor (bFGF) induces premature 0-2A progenitor differentiation. Conversely, the effects of PDGF and bFGF in combination are indefinitely to stimulate proliferation and delay differentiation until such time as the growth factor environment changes whereupon oligodendrocyte maturation promptly occurs. In this way, the intrinsic clockwork of the $0-2 \mathrm{~A}$ progenitor can be suspen$\mathrm{ded}^{9}$. The signals that interact in determining growth, migration and proliferation in $0-2 \mathrm{~A}$ progenitors and their progeny have not been fully defined but they seem to depend neither on alteration in the expression of the PDGF receptor, its density on the cell surface, increased intracellular signals involving calcium, nor changes in the expression of early immediate genes ${ }^{10-11}$.

Differentiating oligodendrocytes express the 04 antigen; other myelin proteins and glycolipids are produced in sequence by the developing oligodendrocyte and concentrated at different sites on the cell process. Galactocerebroside is synthesised early and distributed throughout the cell membrane; the major component myelin basic protein is polarised on the cytoplasmic surface, and the quantitatively minor proteolipid protein and CNP-ase are found on external parts of the cell process; myelin associated glycoprotein is an adhesion molecule found at the leading edge of the myelin membrane that first contacts the axon and together with other, as yet unidentified, molecules ensures tight lamellation of the membrane as the myelin sheath forms.

Neonatal $0-2 \mathrm{~A}$ progenitors that continue to populate the myelinating optic nerve encounter an altered growth factor environment and under the influence of cilary neurotrophic factor, also produced by protoplasmic or type 1 astrocytes, differentiate into type 2 astrocytes; these contact the nodes of Ranvier and so complete the oligodendrocyte myelin unit required for saltatory conduction $^{12}$.

Most of the controversy in developmental glial neuroscience hinges on the status of the type 2 astrocyte. This cell is believed to represent the alternative differentiation stage of the $0-2 \mathrm{~A}$ progenitor and, in vitro, stains positively for A2B5 and GFAP; it corresponds in vivo to the fibrous astocyte of Cajal. However, in long term culture it has an unstable phenotype and begins to look rather like a type 1 astrocyte in terms of ion channel profile, morphological appearance and antigen expression; and it cannot be identified in vivo. ${ }^{3}$ Those who remain uncertain about the type 2 astrocyte also question the concept of bipotentiality in the macroglial arrangements of the neonatal and adult nervous system. Using evidence for cell division (thymidine incorporation) and antigen profile to define oligodendroblasts, astroblasts or bipotential cells, the conclusion has 
been reached that $70 \%$ of cells in the optic nerve are astroblasts in the first post natal week, $70 \%$ oligodendroblasts at weeks two and three, and that there are no progenitors ${ }^{13}$. Furthermore, the use of marker dyes to reveal in vivo glial arrangements in the optic nerve only show astrocytes that contact nodes of Ranvier, blood vessels and the pial surface suggesting that the morphological arrangements claimed for types 1 and 2 astrocytes may be in vitro artefacts $^{14}$.

Those who take the view that the type 2 astrocyte is a distinct entity argue from the viewpoint that morphological and cell surface phenotype are critically dependent on the markers used to define each cell type, many of which, with experience, have been shown not to be cell or lineage specific. According to this analysis, the oligodendroblast and the $0-2 \mathrm{~A}$ progenitor are one and the same cell ${ }^{9}$.

Clonal analysis of the developing rodent cortex further complicates ideas on glial development and indicates that a cell type exists that in some respects resembles the elusive type 2 astrocyte but differs from it and all other macroglial cells in distribution, morphology and cell surface antigen profile (GFAP and A2B5 positive but 04 and GalC negative, $\left.{ }^{15}\right)$.

Several lines of evidence suggest that the adult optic nerve and other parts of the nervous system can be repaired following oligodendrocyte depletion and demyelination. Since differentiated oligodendrocytes cannot be replaced, the assumption has to be made that repair is effected by $0-2 \mathrm{~A}$ progenitors which retain the ability to proliferate and migrate in response to platelet derived and fibroblast growth factors ${ }^{16}$. The status of the adult progenitor is important since manipulating growth factor conditions and limiting the processes involved in tissue injury might create an environment in which areas of demyelinated axons would be reconstituted by glial cells and remyelinated. A remyelinating cell has been identified in the acute demyelinating lesions seen in patients with multiple sclerosis ${ }^{17}$, in immunological and virus induced demyelination in mice ${ }^{18,19}$ and in experimental anti-galactocerebroside antibody mediated demyelination of the cat optic nerve ${ }^{20}$.

One other feature of glial neurobiology that requires consideration is the inhibitory effects of glia on neuronal regeneration. Although axons will grow across sheets of asrocytes in culture, this does not reliably model glial arrangements in vivo; three dimensional cultures of astrocytes present a physical barrier that regenerating axons will not cross in contrast to a matrix assembled from Schwann cells ${ }^{21}$. The astrogliosis that is a feature of chronic demyelinating plaques presents a physical barrier to axonal penetration, limiting access of other regenerative elements into chronic lesions. In addition, mature oligodendrocytes actively inhibit axonal regeneration ${ }^{22,23}$. The molecular basis for inhibition has been partially characterised and, experimentally, oligodendrocyte disinhibition can be achieved using monoclonal antibodies which block the relevant cell surface molecules permitting increased but nevertheless limited growth following axo- nal injury both in vitro and in vivo ${ }^{24-26}$. In neither situation is axonal regeneration comparable to that seen in the peripheral nervous system or when there is contact with Schwann cells.

Environmental factors are not, however, unique determinants of axonal regeneration; cells obtained from embryonic tissue grow better than the axons of adult neurones and human nerve cells will cover distances that permit full connectivity in the rat brain ${ }^{27}$ whereas equivalent regeneration in the human brain would represent failure of repair. Differences in the extent to which embryonic and adult growth cones penetrate their surrounding matrix are thought to depend directly on local secretion of proteases, which is increased by FGF and interleukin-1, and indirectly on the availability of serine protease inhibitors. The astrocyte factor that inhibits regeneration of adult axons has a high concentration in gray matter and shows regional specificity, enhancing growth at one site whilst inhibiting another ${ }^{28}$.

In these ways, intrinsic and presumably genetically determined growth schedules, together with environmental cues interact in determining growth and limiting regeneration in the adult nervous system. Loss of regeneration seems to be the price paid by the adult nervous system for achieving stable but enormously complex glial-neuronal arrangements.

\section{IN VITRO STUDIES ON THE IMMUNOBIOLOGY OF GLIA}

Studying oligodendrocytes and astrocytes in vitro allows provisional conclusions to be reached concerning mechanisms of myelin injury that occur in the intact central nervous system. Immune responses developing in the optic nerve, or other parts of the central nervous system, depend upon increased permeability of the blood brain barrier. The available evidence suggests that an important early event is alteration in expression of cell surface adhesion molecules associated with $\mathrm{T}$ lymphocyte and macrophage activation; at this point, the circulation slows and with the establishment of electrostatic forces, vascular addressins are expressed, cytokines released, endothelial matrix degrades, and the barrier opens bringing an array of potentially pathogenic inflammatory cells and mediators to the abluminal surface of blood vessels ${ }^{29,30}$. Although activated $\mathrm{T}$ cells could themselves be directly cytotoxic for some component of the oligodendrocyte myelin unit, it seems more likely that they influence demyelination indirectly either by inducing gamma interferon, which activates macrophages and microglia, or by the providing local help for B lymphocytes and antibody production.

Incubating oligodendrocytes with normal serum, mimicking the consequences of breakdown in the blood brain barrier, causes antibody independent classical pathway activation of complement and cell lysis ${ }^{31,32}$. Recent studies $^{33,34}$ have defined two molecular defects on the cell surface. An as yet unidentified ligand binds $\mathrm{Cl}$ and leads to the conversion of $\mathrm{C} 3$ stimulating assembly of the membrane attack complex on the oligodendrocyte cell surface. 
Nucleated cells normally possess a variety of regulatory proteins that protect from membrane attack complex formation, one of which (CD59) is lacking from the surface of neonatal (rat) optic nerve and cortical oligodendroglia.

Membrane attack complexes forming on the cell surface, increase membrane permeability and cause a rise in intracellular calcium. Indeed the subsequent fate of the cell seems to depend on whether or not this rise in calcium is contained, since any agent which increases calcium permeability, including $\mathrm{T}$ cell derived perforins or calcium ionophores, precipitates the same cascade of potentially lethal events ${ }^{35}$.

Following a modest rise in intracellular calcium, oligodendrocytes resist cell lysis by a repair process in which the membrane pores are shed from the cell surface as vesicles, restoring membrane stability and cytoplasmic homeostatis ${ }^{36}$; vesicular repair is stimulated by changes in intracellular calcium. Complement activation has a further consequence in that production of the macrophage chemotactants and activators $\mathrm{C} 3 \mathrm{a}, \mathrm{C} 3 \mathrm{~b}$ and $\mathrm{C} 5 \mathrm{a}$, are sufficient in vitro to cause macrophage or microglial adherence to oligodendrocytes but only if these phagocytotic cells have been gamma inteferon activated, or the oligodendrocytes are opsonised by any antibody that binds to the cell surface $^{34,37}$. Vesicles, similar to those released from oligodendrocytes injured by pore forming agents in vitro, are found in the spinal fluid of patients with multiple sclerosis and the membrane attack complexes of complement can be detected immunocytochemically within lesions, demonstrating that complement activation occurs in patients with demyelinating disease $\mathrm{e}^{36,38}$.

Thus a hypothesis for the pathogenesis of human brain inflammation and demyelinating disease, based on in vitro studies of cells populating the optic nerve, is that activated $T$ cells damage the blood brain barrier leading to an accumulation of soluble and cellular immune mediators on the abluminal surface of cerebral vessels. Complement is fixed and activated on the oligodendrocyte cell surface and this results either in cell lysis or vesicular repair. Vesicles expose determinants not normally seen by the immune system and are immunogenic. Tolerance is lost and secondary immune responses occur resulting in local production of anti-oligodendrocyte or myelin antibodies, especially after repeated cycles of injury and repair ${ }^{39}$. The conditions are then established for macrophages to contact and degrade myelin or oligodendrocytes. This is the morphological hallmark of inflammatory disease in the central nervous system.

The dissociation between blood brain barrier permeability and myelin injury is supported by the observation that a high proportion of patients with optic neuritis, and demyelination affecting other parts of the nervous system, show retinal sheathing; this arises from increased vascular permeability but is occurring at a site where there are no myelinated axons $\mathrm{s}^{40,41}$

Further evidence is provided by magnetic resonance imaging in which $\mathrm{T} 2$ weighted signals reflect the water content of regions under scrutiny. Almost every patient with clinically definite multiple sclerosis has periventricular lesions and $>90 \%$ also show discrete white matter abnormalities; both correspond to areas of histological damage. Lesions can be demonstrated in the optic nerve, brain stem and spinal cord in patients with clinical involvement at these sites ${ }^{42,43}$. In optic neuritis, the nerve appears swollen with a focal increase in magnetic resonance signal; inflammation within the intracanalicular portion and long lesions are associated with delayed or incomplete recovery of vision ${ }^{44}$. These may be the cases in whom treatment with oral or intravenous corticosteroids, early in the course, could favourably influence the visual prognosis. Correlations between magnetic resonance images, the evolution of symptoms, and clinical neurophysiology show that the visual deficits in optic neuritis arise from altered blood brain barrier permeability and conduction block, without demyelination or axonal degeneration necessarily having occurred ${ }^{45}$

Gadolinium enhancement provides information on permeability of the blood brain barrier. Serial studies of individual patients have established important principles about the dynamics of plaque formation. The earliest change seen in an evolving lesion is an increase in blood brain barrier permeability ${ }^{46}$; new lesions are first recognisable as areas of gadolinium enhancement which last up to four weeks and precede the onset of $\mathrm{T} 2$ weighted changes, and symptoms, by approximately two weeks ${ }^{47}$. As the lesion evolves, persistent enhancement is seen to occur as a ring around the edge together with a uniform increase in signal. Subsequently the enhancement may cease, leaving a fixed $\mathrm{T} 2$ weighted abnormality; the lesion may disappear or reactivate with ring enhancement, the cycles lasting about eight weeks ${ }^{48}$. The stable periventricular high signal abnormalities correlate best with areas of persistent demyelination, and depend on the increased water content associated with astrocytosis. New, evolving and resolving lesions may be seen in an individual patient; serial studies show that new or reactivated imaging abnormalities occur perhaps 15 times more frequently than clinical episodes.

\section{OPTIC NEURITIS}

The symptoms of optic neuritis (pain on eye movement, followed by blurred vision), evolve over hours or days, the nadir of the visual loss varying from slight blurring to blindness. Some patients notice selective involvement of colour vision, usually in the red range; others describe disturbances of visual perception with persistence of images or flashes of light (phosphenes) provoked by eye movement. Pain disappears after a few days; vision improves rapidly at first and then more slowly, full recovery usually taking several months. About $90 \%$ of patients consider that vision eventually returns to normal but objectively up to $50 \%$ have persistent visual defects, and colour perception often remains impaired. The symptoms of bilateral optic neuritis do not differ from unilateral disease but the two eyes may be differentially affected and there is a lower recurrence risk.

Optic neuritis is a common presenting feature of 
multiple sclerosis and the visual pathway is invariably involved at some stage. This leads to a high level of anxiety in the informed patient that an episode of optic neuritis may be the first manifestation of a recurrent and potentially disabling demyelinating illness. In a recent series of 118 patients, eligible for inclusion in a prospective study of isolated optic neuritis, 21 were retrospectively thought to have had multiple sclerosis at presentation; in 14 the visual evoked response was normal despite the complaint of blurred vision and five of these were considered to have hysterical loss of vision; no conclusions could be reached in 19 patients, and 16 were later shown to have another disorder. Within 3.5 years of the initial episode, $60 \%$ of the remaining 48 patients had experienced manifestations of demyelinating disease affecting other parts of the nervous system ${ }^{49}$.

Age at presentation correlates with the recurrence risk for multiple sclerosis. Optic neuritis is commonly bilateral in children, and new episodes of demyelination seldom occur. Bilateral simultaneous optic neuritis is less common in adults than children but also carries a low risk of multiple sclerosis; the likely explanation is that in both groups, this syndrome represents an anatomically restricted form of acute disseminated encephalomyelitis. One other age related aspect of optic neuritis is that clinical recovery in children is often associated with a return to normal both in latency and amplitude of the visual evoked response ${ }^{50}$.

The risk of recurrence is highest in the first five years after optic neuritis but the proportion of cases with widespread demyelination continues to increase thereafter. Thirtyfive percent of patients observed serially in a North American cohort had developed multiple sclerosis by 1977 compared with $58 \%$ in $1985^{51}$; a recurrence risk of $40 \%$ was observed in a group of patients studied serially in southern Sweden over 15 years $^{52}$. Life table analysis, compensating for varying length of follow-up, gives estimates of 38\%-78\% depending on the geographical location and choice of actuarial time point.

The study of optic neuritis in children and adults raises an issue which is fundamental to the study of human demyelinating disease; why is recovery unstable in adults and associated with persistent disability and physiological abnormalities, whereas in children there is functional and (presumably) structural repair?

The extent to which brain or optic nerve demyelinating lesions damage can be repaired depends on severity and duration of the initial damage, whether the insult is repeated, and the regenerative capacity of the injured parts. Damage limitation assists regeneration by reducing the requirements for repair. In the case of myelinated fibres, injury starts with cellular penetration of the blood brain barrier; demyelination is mediated by phagocytic cells and conditioned by a complex interplay of extrinsic and genetic factors. The contribution made by each aetiological factor differs in the monophasic demyelinating disorders of childhood (which are usually virus induced and not influenced by genetic background) and in multiple sclerosis (in which viral triggers operate against an influential genetic background). Athough their interaction determines the dynamics of tissue injury, another important factor is the regenerative potential of the affected parts and the extent to which this capacity is compromised by the disease process at a cellular level. Thus the possibilities arise that the availability of $0-2 \mathrm{~A}$ progenitors (or differentiated oligodendrocytes) capable of remyelination is greater in children than adults, and that the growth factor environment in which these cells are seeking to restablish contact with naked axons may favour repair in developing white matter by contrast with the mature nervous system. There may be important factors in the pathogenesis of viral demyelinating diseases in children and the relapsing disorders of adults that differentially target progenitors and so eliminate the potential for remyelination of fully evolved lesions.

\section{REFERENCES}

1. Perry VH and Gordon S: Macrophages and microglia in the nervous system. Trends Neurosci 1988, 11: 273-7.

2. Bjorklund A ed: Transplantation in the nervous system. Trends Neurosci 1991, 14: 319-88.

3. Cameron RS and Rakic P: Glial cell lineage in the cerebral cortex: a review and synthesis. Glia 1991, 4: 124-37.

4. Misson J-P, Takahashi T, Caviness VS: Ontogeny of radial and other astroglial cells in the murine cerebral cortex. Glia1991, 4: 138-48.

5. Raff MC, Miller RH, Noble M: A glial progenitor that develops in vitro into an astrocyte or an oligodendrocyte depending on culture medium. Nature 1983, 303: 390-2.

6. Miller RH, ffrench Constant C, Raff MC:. The macroglial cells of the rat optic nerve. Ann Rev Neurosci 1989, 12: 517-34.

7. ffrench Constant C, Raff MC: Proliferating bipotential glial progenitor cells in adult rat optic nerve. Nature 1986, 319: 499-502.

8. Noble M, Murray K, Stroobant P, Waterfield MD, Riddle P: Platelet derived growth factor promotes division and motility and inhibits premature differentiation of the oligodendrocyte/type 2 astrocyte progenitor cell. Nature 1988, 333: 560-2.

9. Noble M: Points of controversy in the $0-2 \mathrm{~A}$ lineage: clocks and type 2 astrocytes. Glia 1991, 4: 157-64.

10. Hart IK, Richardson WD, Heldin $\mathrm{CH}$, Westermark B, Raff MC: PDGF receptors on cells of the oligodendrocyte-type 2 astrocyte (0-2A) cell lineage. Development 1989, 105: 595-604.

11. Hart IK, Richardson WD, Bolsover SR, Raff MC: PDGF and intracellular signalling in the timing of oligodendrocyte differentiation. J Cell Biol 1989, 109: 3411-17.

12. Miller RH, Fulton BP, Raff MC: A novel type of glial cell associated with nodes of Ranvier in rat optic nerve. Eur $J$ Neurosci 1989, 1: 172-80.

13. Skoff RP, Knapp PE: Division of astroblasts and oligodendroblasts in postnatal rodent brain: evidence for separate astrocyte and oligodendrocyte lineages. Glia 1991, 4: 165-74.

14. Butt AM and Ransom BR: Visualisation of oligodendrocytes and astrocytes in the intact rat optic nerve by intracellular injection of Lucifer yellow and horseradish peroxidase. Glia 1991, 2: 470-5.

15. Goldmann JE and Vaysse PJ-J: Tracing glial cell lineages in the mammalian forebrain. Glia 1991, 4: 149-56.

16. Wolswijk $\mathrm{G}$ and Noble $\mathrm{M}$ : Identification of an adult specific glial progenitor cell. Development 1989, 105: 387-400. 
17. Prineas JW, Kwon EE, Goldenberg PZ, Ilyas AA, Quarles RH, Benjamins JA, Sprinkle TJ: Multiple Sclerosis. Oligodendrocyte Proliferation and Differentiation in fresh lesions. Lab Invest 1989, 61: 489-503.

18. Armstrong R, Friedrich VL, Holmes KV, Dubois-Dalcq M: In vitro analysis of the oligodendrocyte lineage in mice during demyelination and remyelination. J Cell Biol 1990, 111: 1183-95.

19. Godfraind C, Friedrich VL, Holmes KV, Dubois-Dalcq M: In vivo analysis of glial cell phenotypes during viral demyelinating disease in mice. J Cell Biol 1989, 109: 2405-16.

20. Carroll WM, Jennings AR, Mastaglia FL: The origin of remyelinating oligodendrocytes in antiserum-mediated demyelinative optic neruropathy. Brain 1990, 113: 953-73.

21. Fawcett JW and Housden E: The effects of protease inhibitors on axon growth through astrocytes. Development 1990 , 109: 59-66.

22. Fawcett JW, Rokos J, Bakst I: Oligodendrocytes repel axons and cause axonal growth cone collapse. J Cell Sci 1989, 92: 93-100.

23. Fawcett JW, Housden E, Smith-Thomas L, Myer RL: The growth of axons in 3 dimensional astrocyte cultures. Dev Biol 1989, 135: 449-58.

24. Caroni P and Schwab ME: Two membrane protein fractions from rat central myelin with inhibitory properties for neurite growth and fibroblast spreading. J Cell Biol 1988, 106: 1281-88.

25. Caroni $P$ and Schwab ME: Antibody against myelin associated inhibitor of neurite growth neutralises non-permissive substrate properties of CNS white matter. Neuron 1988, 1: 85-96.

26. Schnell $\mathrm{L}$ and Schwab ME: Axonal regeneration in the rat spinal cord produced by an antibody against myelin associated neurite growth inhibitors. Nature 1990, 343: 269-72.

27. Wiktorin K, Brundin P, Gustavi B, Lindvall O, Bjorklund A: Reformation of long axon pathways in adult rat central nervous system by human forebrain. Nature 1990, 347: $556-8$.

28. Stern CD, Sisodiya SM, Keynes RJ (1986): Interactions between neurites and somite cells: inhibition and stimulation of nerve growth in the chick embryo. J Embryol Exp Morph 1986, 91: 209-26.

29. Male, DK, Pryce G, Hughes CCW, Lantos PL: Lymphocyte migration into the brain modelled in vitro: control by lymphocyte activation, cytokines and antigen. Cell Immunol 1990; 127: 1-11.

30. Hickey WF: Migration of hematogenous cells through the blood brain barrier and the initiation of CNS inflammation. Brain Pathol 1991, 1: 97-105.

31. Scolding NJ, Morgan BP, Houston WAJ, Campbell AK, Linington C, Compston DAS: Normal rat serum cytotoxicity against syngeneic oligodendrocytes: complement activation and attack in the absence of antimyelin antibodies. $J$ Neurol Sci 1989, 89: 289-300.

32. Wren DR and Noble M: Oligodendrocytes and adult specific 0-2A progenitor cells are uniquely susceptible to the lytic effects of complement in the absence of antibody. Proc Natl Acad Sci 1989, 86: 9025.

33. Wing MG, Zajicek JP, Seilly DJ, Compston DAS, Lachmann PJ: Inhibition of antibody-dependent classical pathway activation by oligodendrocytes using CD59. Immunology 1992 (in press).

34. Zajicek JP, Wing M, Lachmann PJ, Compston DAS: Oligodendrocyte interactions with human serum - the simultaneous demonstration of antibody independent classical pathway complement activation and abnormal sensitivity to terminal complement attack. J Neuro Sci 1992 (in press).

35. Scolding NJ, Morgan BP, Campbell AK, Compston DAS:
The role of calcium in rat oligodendroctye injury and repair. Neurosci Lett 1992 (in press).

36. Scolding NJ, Morgan BP, Houston WAJ, Linington C, Campbell AK, Compston DAS (1989). Vesicular removal by oligodendrocytes of membrane attack complexes formed by complement. Nature 1989 339, 620-2.

37. Scolding NJ and Compston DAS: Oligodendrocye macrophage interactions in vitro triggered by specific antibodies. Immunology 1991, 72: 127-32.

38. Compston DAS, Morgan BP, Campbell AK, Wilkins P, Cole G, Thomas ND, Jasani B: Immunocytochemical localisation of the terminal complement complex in multiple sclerosis. Neuropath and Appl Neurobiol 1989, 15: 307-316

39. Compston DAS, Scolding NJ, Wren DR, Noble M: The pathogenesis of demyelinating disease: insights from cell biology. Trends Neurosci 1991, 14: 175-82.

40. Shaw PJ, Smith NM, Ince PG, Bates D: Chronic periphlebitis retinae in multiple sclerosis. A histological study. $J$ Neurol Sci 1987, 77: 142-52.

41. Lightman S, McDonald WI, Bird AC, Francis DA, Hoskins A, Batchelor JR, Halliday AM: Retinal venous sheathing in optic neuritis. Its significance for the pathogenesis of multiple sclerosis. Brain 1987, 110: 405-14.

42. Miller DH, McDonald WI, Blumhardt LD, du Boulay GH, Halliday AM, Johnson G, Kendall BE, Kingsley DPE, MacManus DG, Moseley IF, Rudge P, Sandercock PAG: Magnetic resonance imaging in isolated spinal cord syndromes. Ann Neurol 1987, 22: 714-23.

43. Miller DH, Newton MR, van der Poel JC: Magnetic resonance imaging of the optic nerve in optic neuritis. Neurology 1988, 38: 175-9.

44. Youl BD, Miller DH, MacManus DG, Moore SG, Barnes D, Kermode AG, Hawkins CP, Kendall BE, Moseley IF, Tofts PS, Thompson AJ, McDonald WI: Optic nerves show gadolinium enhancement in early optic neuritis. Neuroradiol 1991, 33 [Suppl]: 129-30.

45. Youl BD, Turano G, Miller DH, Towell AD, MacManus DG, Moore SG, Jones SJ, Barrett G, Kendall BE, Moseley IF, Tofts PS, Halliday AM, McDonald WI: The pathophysiology of acute optic neuritis: an association of gadolinium leakage with clinical and electrophysiological deficits. Brain 1992, 114: 2437-50.

46. Kermode AG, Thompson AJ, Tofts P, MacManus GG, Kendall BE, Kingsley DPE, Moseley IF, Rudge PR, McDonald WI: Breakdown of the blood brain barrier precedes symptoms and other MRI signs of new lesions in multiple sclerosis. Brain 1990, 113: 1477-89.

47. Miller DH, Rudge PR, Johnson G, Kendall BE, MacManus DG, Moseley IF, Barnes D, McDonald WI: Serial gadolinium enhanced magnetic resonance imaging in multiple sclerosis. Brain 1988, 111: 927-39.

48. Koopmans RA, Li DKB, Oger J J-F, Mayo J, Paty DW: The lesions of multiple sclerosis: imaging of acute and chronic stages. Neurol 1989, 39: 959-63.

49. Sanders EACM and van Lith GHM: Optic neuritis, confirmed by visual evoked response, and the risk for multiple sclerosis: a prospective study. J Neurol Neurosurg Psych 1989, 52: 799-800.

50. Parkin PJ, Hierons R, McDonald WI: Bilateral optic neuritis: a long term follow-up. Brain 1984, 106: 951-64.

51. Rizzo JF and Lessell S: Risk of developing multiple sclerosis after uncomplicated optic neuritis: a long term prospective study. Neurology 1988, 38: 185-90.

52. Sandberg-Wollheim S, Bynke H, Cronqvist S, Holtas S, Platz P, Ryder LP: A long term prospective study of optic neuritis: evaluation of risk factors. Ann Neurol 1990, 27: 386-93. 